\title{
Quaderni
}

QUADERNI Communication, technologies, pouvoir

97 | Automne 2018

Néo-libéralisme(s). Réseaux et formes des

mobilisations en France

\section{L'engagement dans un think tank néo-libéral}

Entretien avec Gaspard Koenig, président de GénérationLibre

\section{Kevin Brookes}

\section{(2) OpenEdition}

Journals

Édition électronique

URL : http://journals.openedition.org/quaderni/1285

DOI : 10.4000/quaderni.1285

ISSN : 2105-2956

Éditeur

Les éditions de la Maison des sciences de l'Homme

Édition imprimée

Date de publication : 5 octobre 2018

Pagination : 57-67

\section{Référence électronique}

Kevin Brookes, «L'engagement dans un think tank néo-libéral », Quaderni [En ligne], 97 | Automne 2018, mis en ligne le 05 octobre 2020, consulté le 04 janvier 2021. URL : http://journals.openedition.org/ quaderni/1285; DOI : https://doi.org/10.4000/quaderni.1285 


\section{$D$ ossier}

\section{L'engagement dans un}

think tank néo-libéral.

\section{Entretien avec}

Gaspard Koenig, président de

\section{Par Kevin Brookes}

Doctorant en science politique Laboratoire PACTE Science-Po Grenoble
Comment s'engage-t-on pour les idées néo-l ibérales en France? En tant que directeur du think tank GénérationLibre et philosophe engagé dans le débat public, Gaspard Koenig nous offre une vue de l'intérieur du mouvement néo-libéral en France. Ancien élève de l'ENS, agrégé de philosophie, écrivain, ancienne plume de ministre, candidat aux élections législatives de 2012 pour les Français de l'étranger, son cheminement professionnel et intellectuel est riche d'enseignements. Tout d'abord, il témoigne de l'existence de plusieurs branches du néo-libéralisme. Les idées qui animent Gaspard Koenig proviennent de ses lectures en philosophie politique davantage que de la lecture d'économistes théoriciens du marché. Son néolibéralisme modéré se distingue de traditions concurrentes qui font de l'État la source de tous les maux de la société. Il s'inscrit, à l'inverse, dans une tradition libérale qui attribue à l'État un rôle d'arbitre s'assurant que chacun ait la capacité d'être autonome et de choisir son mode de vie. Dans cet entretien, il nous expose les raisons qui l'ont poussé à défendre et à créer un think tank, GénérationLibre, pour s'assurer de la promotion de ces idées (plutôt que des répertoires d'action concurrents). Il explique son fonctionnement et les raisons qui l'ont poussé à privilégier le registre de l'expertise par rapport à celui de l'" intellectuel total ». Il expose sa démarche inspirée de l'expérience des think tanks au Royaume-Uni et du penseur néo-libéral Friedrich Hayek, visant à convaincre un «public éclairé » composé d'intermédiaires de pensée. L'objectif est pour le philosophe de changer le climat d'opinion régnant dans la société en tenant un discours respecté par les élites. Pour ce faire, il privilégie la production d'expertise à 
disposition des décideurs publics, ainsi que l'établissement de réseaux de personnes partageant sa vision du libéralisme. Son témoignage révèle également que, si les mobilisations en faveur $d u$ néo-libéralisme se développent et revêtent des formes comparables à celles observées dans les pays qui ont donné naissance à cette idéologie, elles demeurent contraintes par plusieurs facteurs institutionnels propres au contexte français.

\section{Je voudrais revenir avec vous sur votre} cheminement vers les idées libérales. Votre parcours est désormais relativement connu, mais on ignore comment vous en êtes venu à les défendre. Comment découvre-t-on les idées libérales en France?

C'est une bonne question. D'abord, j'étais avant tout philosophe et j'ai fait une maîtrise sur Deleuze et mon DEA sur Condillac. J'étais donc familier de la philosophie française dite classique. J'ai découvert le libéralisme par différents biais. J'étais allé vivre un an à New York où j'ai étudié à l'Université de Columbia et j'ai eu quelques cours sur les idées libérales notamment. J'ai également commencé à lire The Economist, j'ai découvert Condillac, qui est aussi un libéral, plus ou moins, en tout cas dans la tradition physiocratique. Et puis, j'ai lu Tocqueville et Revel. C'est un peu cette combinaison-là.

Quand je suis revenu en France, après cette année newyorkaise, après ces cours, ces lectures, et aussi à avoir fréquenté ce milieu très différent des agrégés français, j'ai continué à creuser cette voie et lire The Economist qui est vraiment un repère important pour moi ; tous les samedis depuis dix ans je lis The Economist. La ligne qu'ils ont - à quelques détails près - est très similaire à celle de GenerationLibre, le think tank que je préside et qui défend un libéralisme très classique, mais très affuté sur les sujets les plus modernes et très réputé sur les aspects technologiques.

Est-ce que l'on peut dire que vous êtes arrivé au libéralisme économique, ce que certains appellent le néo-libéralisme, par le libéralisme politique classique en fait ?

Complètement. Je suis arrivé au libéralisme par la philosophie, c'est sûr, en lisant Condillac, Tocqueville et Revel qui sont les trois premiers auteurs que j'ai lus ensemble concernant la question des libertés de l'individu. J'ai découvert le marché chemin faisant, d'abord parce que cela n'a jamais été ma tasse de thé et les questions des mécanismes de marché ne sont pas celles qui m'excitent le plus. Mais je comprends combien cela peut être important en tant que reflet de la conception des libertés. Tout part de la conception de la liberté politique, en effet, de la conception de l'individu grosso modo et de son indépendance, de son autonomie et de ses capacités de choix. Ensuite, il y a différents idéaux économiques qui en découlent.

Justement, parce que vous parlez de plusieurs libéralismes économiques, il y a différents vocables pour désigner vos idées et celles de GenerationLibre. Certains parlent de néolibéralisme, d'autres d'ultra-libéralisme, d'autres encore de libertarianisme. Finalement, quel vocable serait le plus adéquat pour qualifier vos idées ?

D'abord, je crois qu'il y a quelque chose d'im- 
portant chez les libéraux : ils n'ont pas de cases très fixes. C'est inhérent au progrès de cette pensée qui est libre vis-à-vis d'elle-même et qui, contrairement à d'autres pensées beaucoup plus dogmatiques, est toujours en mouvement, et qui doute - ce qui est mon cas. Condillac, c'est à la fois quelqu'un qui écrit un traité de commerce, qui était pour l'ouverture des frontières, les échanges, etc. et c'était aussi quelqu'un qui a écrit un traité contre les systèmes. Il dit lui-même que la philosophie fonctionne dans un système, et Descartes et Spinoza analysaient ça, et c'est parce que ce sont des systèmes clos que cela ne peut être que faux. Je ne dis pas cela pour éviter de répondre à votre question, c'est pour montrer que sur une base libérale classique, il y a ensuite beaucoup de chemins et d'embranchements. Je n'ai pas tout résolu et je n'ai pas de mot pour qualifier tout ça.

Alors, le mot « Ultra-libéralisme » que vous utilisez ne permet pas de caractériser le fond d'une pensée, c'est juste une insulte en fait, cela n'a pas d'intérêt. « Néo-libéralisme », j'ai souvent pensé que c'était la même chose, mais j'ai été convaincu par la lecture de Serge Audier, ainsi que par celle de Foucault. Cela montre qu'il existe bien une réalité politique et historique assez profonde et de ce point de vue-là, je dois dire que je me sens plutôt disons ordo-libéral, que néo-libéral à la Friedman. Il y a eu toujours quelque chose qui m'a un peu dérangé dans le tournant américain de la Société du Mont-Pèlerin, depuis que les ordolibéraux se sont effacés et depuis qu'il y a une hégémonie des économistes sur le mouvement et surtout l'hégémonie des idées de marché. Je trouve qu'on en est toujours là, et que la Société du Mont-Pèlerin traîne encore ces idées-là, alors que le libéralisme est plus complexe et plus touffu. Ils ont réduit le libéralisme à une doctrine très systématique. Par ailleurs, il y a chez eux une opposition totale au rôle du gouvernement - pas chez Friedman - qui est assez paradoxale, parce que les libéraux comme Adam Smith ont inventé l'État moderne. Ils ont inventé la notion même Foucault le dit - de gouvernementalité. On peut le leur reprocher, mais en tout cas, le libéralisme s'est construit avec l'État, et avec l'idée d'une régulation et d'un gouvernement. Donc opposer le libéralisme à l'État est très bizarre, très récent et très marginal dans l'histoire de la doctrine quand on la regarde bien. Je pense que c'est parce que l'État est devenu si envahissant et si pénible que l'on passe notre temps à lutter contre des lois stupides. Mais on a tendance à généraliser théoriquement en disant que tout gouvernement est condamnable, alors que ce n'est pas du tout l'essence de cette doctrine, qui au contraire imaginait un rapport direct de l'État à l'individu (en brisant les corporations, etc.).

Enfin, sur les « libertariens », je m'intéresse en ce moment aux « libertariens de gauche » ou « bleeding heart libertarians », tout ce que fait le Niskanen Center à Washington par exemple, qui est une sorte d'offset du CATO Institute ${ }^{1}$ en plus modéré, raisonnable, plus fin, et s'intéressant aux problèmes sociaux sans exclure toute régulation d'emblée. Sur le plan plus philosophique, les « libertariens de gauche » s'inscrivent - de Pufendorf à Peter Valentyne - dans l'idée que certes le travail permet de s'approprier des biens, mais en même temps on s'approprie quelque chose qui appartient à tous, alors il y a une logique à compenser la communauté. Ils trouvent donc une justification théorique fondamentale à l'idée 
d'impôts, ils sont d'ailleurs souvent partisans du revenu de base. Il y a cette nouvelle génération de libertariens dits « de gauche », beaucoup plus soucieuse de diversité et d'individualisme que de marché, beaucoup plus intéressée aux problèmes de pauvreté, de prisonniers, etc. parce que c'est facile de montrer que le libertarianisme marche pour les entrepreneurs. Mais l'idée c'est de montrer que le libéralisme marche pour les pauvres, pour les fous. C'est le véritable enjeu. C'est quand on aura montré cela que l'on aura gagné. Montrer qu'il marche pour tout le monde. C'est un peu l'objet de mon dernier ouvrage ${ }^{2}$, pour lequel j'ai voyagé et rencontré des populations pauvres au Pérou, des vendeurs de cannabis au Colorado, des prisonniers en Finlande, des religieux en Inde, etc. Afin de montrer que les solutions libérales apportées à ces enjeux-là pouvaient également fonctionner pour eux. Cette nouvelle génération de libertariens - c'est encore un peu flou, mais je les reconnais - les personnes qui gravitent autour du Niskanen Center, de Van Parijs, de Sam Harris, de Peter Valentyne. Il s'agit d'une toute petite communauté qui n'est pas très structurée, mais où je sens naître une génération très différente de la vieille génération du Mont-Pèlerin.

\section{En fait, vous prenez ainsi vos distances par rapport à l'école autrichienne d'économie ou bien à l'école de Chicago.}

Oui, complètement. Bon je m'intéresse aussi à Friedman, mais je n'ai jamais été fasciné et cela n'a jamais été mon principal centre d'intérêt.

Concernant la promotion des idées, au cours de votre parcours, vous avez décidé de vous lancer dans une campagne pour promouvoir le libéralisme lors des élections législatives en 2012, puis vous avez finalement décidé de fonder votre think tank. Pourquoi choisir ce répertoire d'actions plutôt qu'un autre?

D'abord, je pense qu'il faut distinguer la réflexion théorique qui peut être la mienne et qui le sera davantage dans les années qui viennent, et puis l'engagement dans la Cité via la politique ou via le think tank où j'y défends des idées plus classiques. Je ne peux pas arriver dans le débat public en disant «je suis libertarien de gauche» et simplement donner des directives à suivre. C'est complètement peine perdue. Déjà que c'est assez difficile de défendre le libéralisme. Le libéralisme de GenerationLibre est pour moi relativement classique et transversal. Le débat philosophique que l'on peut avoir reste périphérique par rapport aux activités de GénérationLibre. Je scinde les deux.

Ensuite, sur la raison de mon engagement, j'ai cultivé ces idées un peu tout seul en lisant les grands textes et je travaillais à la BERD [Banque européenne pour la reconstruction et le développement, NDLE] à Londres. J'avais un boulot tout à fait ennuyeux et bien payé. Et puis quand j'ai vu qu'il y avait les premières élections pour les Français de l'étranger, je me suis dit «tiens, voilà une bonne façon d'introduire le libéralisme dans le débat public français qui en manque tellement $»$. Je me suis donc présenté, cela a duré 3 mois et j'ai obtenu 4,5\% aux élections. J'en ai tiré deux conclusions : tout d'abord, alors que les circonstances étaient idéales - à Londres, là où les Français sont à peu près sur cette longueur d'onde, du moins en plus grande proportion qu'en France métropolitaine - et que si je n'obtenais 
que $4,5 \%$, politiquement ce n'était même pas la peine de former un parti libéral en France. Cela n'a vraiment duré que quelques mois et c'est maintenant fini pour toujours. Deuxièmement, j'en ai tiré la conclusion que même si des gens n'étaient pas prêts à voter pour elles, ces idées intéressaient les gens, les médias, d'éventuels sponsors, et cela me plaisait beaucoup de les défendre. J'avais accumulé un réseau, voire une petite expérience durant ces quelques mois de campagne. C'est à partir de là que je me suis demandé comment transformer cela. Et en plus, comme j'allais avoir 30 ans, il fallait que je sorte de mon job bureaucratique. Certains créent des startups, moi je me suis dit, je créé un think tank. Je suis allé voir tous les think tanks en France pour comprendre un peu le marché, ainsi qu'en Angleterre pour comprendre comment ils fonctionnaient et j'ai créé GénérationLibre donc, un peu sur un modèle anglo-saxon, dans la stratégie de financement et le business model, et puis je suis rentré en France pour entrer dans le débat français, encore une fois, sur une plateforme assez large, libertés individuelles, économie de marché, à la fois les sujets économiques et sociétaux, institutionnelles (décentralisation), être contre la $\mathrm{V}^{\mathrm{e}}$ République, tout ce package-là qui était celui de la campagne.

Depuis, je suis convaincu que le combat d'idées est la mère de tous les combats, comme l'a dit Niskanen, un économiste mort il y a 30 ans. Le travail intellectuel que l'on peut mener en France au sein du think tank est beaucoup plus important que si l'on avait un siège de député quelque part. Le think tank influence des générations, des jeunes, qui ensuite iront dans les différents partis. Avoir un parti libéral au pouvoir n'est pas forcément l'objectif pour nous, mais plutôt que les idées pénètrent partout comme cela a été le cas en Angleterre. C'est tout à fait l'expérience de Antony Fisher, un homme d'affaires qui a voulu créer un parti libéral après la Seconde Guerre mondiale. À la place il a créé un think tank, l'Institute for Economic Affairs, qui a irrigué le CPS [Center for Policy Studies, NDLE], devenu le think tank de Thatcher et à l'origine de toutes les grandes réformes des années 1980 et dont l'influence s'est même retrouvée dans le New Labour.

Je reste convaincu de la forme du think tank pour faire de la politique en France de manière intelligente et plus noble, sans s'ennuyer avec des histoires partisanes d'élection notamment. Notre travail est important parce qu'il prépare les élections dans les dix ans à venir... si ça marche je veux dire.

\section{Vous avez donc une grille très hayekienne du point de vue de la diffusion des idées, et cela provient de votre expérience en Angleterre. C'est là-bas que vous avez connu la « forme think tank » ou bien vous la connaissiez déjà en France?}

Oui, c'est bien là-bas en Angleterre que j'ai connu la forme think tank. D'ailleurs, la première levée de fonds pour GenerationLibre a été organisée dans les locaux du CPS, devant le portrait de Margaret Thatcher!

On peut dire que j'ai une vision hayékienne sur le travail des intellectuels en tant qu'intermédiaires de la pensée. Le think tank ne prétend pas faire de la pensée originale. Par exemple lorsqu'on publie 
sur la légalisation du cannabis, on reprend des arguments qui existent déjà partout, mais on les retravaille, on les popularise auprès de l'opinion éclairée, c'est ça un think tank. Ce n'est pas pour aller "grass root», ce n'est pas pour serrer des mains. C'est pour diffuser auprès de l'opinion éclairée, qui ensuite exige du politique qu'il suive ses attentes. La victoire selon moi c'est donc lorsqu'un journaliste pose cette question à un politique sur le revenu universel, la propriété des données, etc. Il n'y a pas d'intérêt à rencontrer les politiques, regarder dans les yeux pour essayer de convaincre, juste par politesse, juste pour exposer l'état du débat. Mais c'est bien en pesant sur l'opinion, via les relais journalistiques, que l'on parvient à transformer les mentalités et à mettre à jour les politiques mises en œuvre.

La forme think tank est très moderne pour la France. Elle est adaptée et permet de sortir de la posture de l'intellectuel solitaire à la française, qui fait tout tout seul et qui réfléchit sur tout. Réfléchir sur tout, c'est légitime, mais l'avantage du think tank c'est qu'on s'entoure d'experts qui vont donner à une idée un peu vague une forme beaucoup plus claire, chiffrée, crédible.

Justement, j'allais vous demander : pourquoi mobiliser le registre de l'expertise au lieu d'aller sur le terrain de l'intellectuel total? Parce que finalement vous êtes philosophe, vous avez écrit des ouvrages sur Deleuze, pourquoi alors ne pas s'engager sur ce terrain-là aussi ?

Je n'ai pas abandonné ce terrain-là pour autant, je continue par exemple à écrire des livres. Mais pour avoir un impact réel et concret dans la société, il faut passer par une institution qui met la main à la pâte, fait du lobbying, du terrain en rencontrant les différents acteurs, ce qui surtout permet de donner chair à des idées un peu générales grâce à son expertise. On aura beau écrire 15 tribunes et 23 livres sur le revenu universel, les gens continueront à dire que ce n'est pas viable, et ce n'est pas complètement faux. Aujourd'hui, il est insupportable de voir ces intellos universels qui tiennent des propos à mille lieues de toute réalité et qui vont continuer à en discuter pendant 300 ans !

En ce sens, l'œuvre d'Alexandre Kojève m'intéresse, concernant l'idée selon laquelle si Platon avait voulu réformer Syracuse, il aurait fallu qu'il passe d'abord par l'administration de Syracuse...

Pour prendre l'exemple du revenu universel, on peut en parler sous l'angle de l'autonomie individuelle, la liberté réelle. Tout ça, c'est gentil, mais ça ne fera qu'alimenter quelques émissions sur France Culture. Alors que nous, nous le faisons avec des experts. Nous lui avons donné une forme très concrète en établissant le coût, l'effet sur les dépenses publiques et la redistribution, le remplacement de tels ou tels impôts, etc. Du coup, même pour les gens qui ne sont pas convaincus par l'idée, elle est passé à travers les filtres des différentes administrations, les cercles de pensée, et les décideurs, qui la prennent au sérieux. Et alors les gens qui ne sont pas convaincus se disent «quand même, c'est peutêtre plus intelligent que ce qu'on fait maintenant, c'est plus pratique, cela ne coûte pas cher». Il peut y avoir toutes sortes de raisons, à partir du moment où le récit est construit, et que cela circule à Bercy, c'est pris au sérieux... et donc je 
pense que c'est beaucoup plus productif de faire ainsi, que d'écrire 15 tribunes.

Cela rétroagit sur les idées, c'est-à-dire que vous ajustez vos idées, ce qui est normal, en fonction de ce que vous voyez par rapport aux problèmes réels que pose l'implémentation. Parce que quand vous parlez avec les experts, vous n'êtes pas obligés d'être expert, mais vous regardez un peu ce qu'ils font, par conséquent vous découvrez plein des problèmes, plein des choses auxquelles vous n'avez jamais pensé. Donc cela rétroagit sur la conception générale de l'idée. Et cet aller-retour entre l'idée et la pratique est essentiel pour moi d'un point de vue philosophique, c'est tout l'objet de mes voyages et du think tank : ne pas rester dans un système uniquement théorique.

\section{Pour le lecteur de Quaderni, comment décri-} riez-vous le quotidien d'un think tank et sa production? Vous parliez de tribunes - c'est ce que vous faites - et des textes d'opinion. Quelles sont les formes de diffusion que vous privilégiez?

Non, certainement il y a des tribunes et des débats médiatiques, mais le fait d'avoir derrière des rapports qui sont solides fait une énorme différence parce que cela donne une vie beaucoup plus longue à l'idée. Une tribune ne dure que 24 heures, alors que si cette tribune est ancrée sur un rapport de 50 pages, celui-ci continuera à vivre et il pourra ressortir des années plus tard, être utilisé dans des programmes de candidats, etc. Le rapport perdure donc bien davantage. Nous avons d'ailleurs le projet de réactualiser les rapports les plus importants du think tank pour que les chiffres restent relativement à jour. Nous participons au débat médiatique, mais je pense que la fonction première du think tank est bien la production de rapports d'expertise, et même si l'impact médiatique n'est pas fort lors de la publication, ceux-ci demeurent dans le temps.

Justement, tout à l'heure vous avez évoqué votre objectif de "viser l'opinion éclairée». Pourquoi ce choix de cibler cette population plutôt que de viser le grand public ? Pourquoi ce choix stratégique?

D'abord, on ne peut pas tout faire, du fait de notre taille réduite, d'où le choix d'une cible en particulier. Ensuite, cela permet d'avoir un message assez raffiné qui correspond à ce que j'aime faire, tout simplement. J'apprécie de moins en moins le fait de devoir simplifier, d'avoir des messages grand public. Et dans l'idéal, il faudrait d'autres personnes qui écriraient à partir de notre travail, en vulgarisant, simplifiant ou en adaptant la forme pour un autre public.

Il faut passer par l'opinion éclairée qui est déjà très large, faire des conférences en province, ce n'est pas seulement les cercles parisiens, c'est plus large. Disons que ça m'intéresse davantage d'avoir un article dans Le Monde que dans Le Parisien. C'est un choix volontaire et quand on peut avoir les deux c'est parfait, on fait les deux. C'est important pour nous d'acquérir une sorte de respectabilité institutionnelle, de graviter dans les pages du Monde, des gens de Paris I, des trucs bien normalisés à la française... C'est assez important d'y être, je pense, pour la qualité de ce qu'on fait, pour ne pas rester trois libertariens dans une cabine téléphonique et cela passe aussi par le fait d'avoir un discours à leur 
niveau. Sinon, si je ne suis qu'un contributeur associé obscur, je ne vais avoir aucune écoute institutionnelle. Ce serait dommage, et cela rapporterait beaucoup moins.

Nous avons fait le choix d'un discours sophistiqué, fin, philosophique, toujours radical, mais qui s'appuie sur l'expertise. La ligne que nous suivons, c'est d'être très radical dans les thèses défendues. On attaque de ce point de vue, mais avec une tonalité, un idéal, et une consistance de production qui permettent d'être vus comme très radicaux, mais aussi très crédible par l'establishment français. Nous voulons être écoutés par l'élite française. C'est très important, parce qu'après cela redescend encore une fois vers l'opinion.

\section{Avez-vous l'impression d'éprouver des diffi- cultés particulières dans le contexte institu- tionnel français au sein duquel les think tanks ne se développent que depuis peu de temps?}

Évidemment. Dans le contexte français d'abord, on a de gros problèmes de financement parce que les think tanks sont mal connus et les plus importants d'entre eux sont financés par l'État ou le CAC 40. Donc on est quasiment dans un terrain vierge, sauf quelques autres institutions qui fonctionnent comme nous. C'est très difficile. Alors qu'en Angleterre ou aux États-Unis, je crois qu'il y a beaucoup plus de place, la perception est différente et davantage de personnes sont prêtes à contribuer. Nous sommes confrontés à un problème culturel difficile à surmonter. Ensuite le fait que peu de think tanks défendent ce que l'on défend n'est pas un atout, c'est plutôt une faiblesse. Nous serions heureux que le marché des idées libérales grossisse, mais il y a peu de popularité de ces idées. Il faudrait davantage d'organisations, mais il n'y en a quasiment pas. C'est sûr qu'avec la ligne qu'on a, on est bien seul.

Enfin, les politiques sont habitués à s'appuyer sur les administrations pour élaborer leurs réformes et leurs programmes, donc très peu d'entre eux prennent les think tanks au sérieux. Ils passent par leurs inspecteurs des finances traditionnels qui établissent des rapports traditionnels. En Angleterre, au contraire, les politiques sont directement en contact avec les think tanks.

C'est intéressant parce que GénérationLibre, contrairement à beaucoup des think tanks américains, les "advocacy tanks», n'a pas de chercheurs et d'experts en interne, et recourt à l'expertise d'universitaires ou de hauts fonctionnaires...

Oui, c'est bien là la dernière difficulté française. En Amérique du Nord, collaborer avec un think tank est bénéfique pour une carrière universitaire. Vous êtes un intello, vous travaillez dans un think tank et ensuite vous revenez au monde universitaire. En France, c'est juste impensable. On le voit d'ailleurs sur le plan pratique, les universitaires ont des statuts précis. Leur collaboration avec un think tank serait considérée comme une déchéance dans leur carrière, ce n'est pas assez réputé.

Il s'agit bien d'un problème institutionnel, qui explique que le recours à des plumes extérieures est indispensable, sinon la production du think tank ne serait pas à la hauteur. C'est également 
une question de moyens bien sûr. Mais recourir à des experts extérieurs est également un atout, parce qu'eux-mêmes ont leurs propres réseaux à activer afin d'étendre la diffusion. C'est aussi intéressant parce que tous nos experts ne partagent pas les mêmes avis, ils ne sont d'ailleurs pas tous complètement libéraux, ils viennent de différents cercles, de la gauche libertaire, du monde des affaires, etc. Ce sont donc des personnes qui ne se seraient peut-être jamais rencontrées en temps normal puisque sociologiquement et culturellement la gauche libertaire et la droite libérale sont complètement séparées, alors que pourtant elles ont beaucoup de choses à se dire. Le fait de les réunir dans une même pièce est alors très agréable, parce que de fortes sympathies naissent chez des gens que tout oppose. Cela permet de créer une vraie petite confrérie, qui sinon n'existerait pas. Avec de gens qui réalisent qu'ils se sentent moins dérangés par le marché ou les lois pour les transsexuels. Tout cela petit à petit crée des ponts, et cela c'est important aussi.

\section{D'accord. C'est donc aussi cela le rôle d'un think tank : créer des réseaux.}

Oui, tout à fait. Je vois d'ailleurs des gens de GenerationLibre qui s'entraident dans tels ou tels colloques, business, etc. selon les secteurs. Ça me fait toujours extrêmement plaisir parce que cela suscite des collaborations concrètes.

Une question pour conclure cet entretien : comment jugeriez-vous de l'influence des actions que vous avez menées ces dernières années? Pensez-vous avoir davantage pesé dans le débat public avec cette forme d'engagement par rapport à celle que vous aviez

\section{choisie auparavant?}

Sur le débat public je suis très content, parce que nos idées ont largement été commentées, reprises, analysées. Nous avons désormais une plateforme médiatique. À chaque nouveau rapport, nous avons des échos un peu partout et nous sommes bien identifiés. Les gens ont compris, après un long combat, que la sorte de libéralisme qu'on représentait n'était pas celui qu'ils fantasmaient. Les préjugés sur les méchants capitalistes patronaux, et l'usage de qualificatifs comme «les banquiers » et autres, c'est du passé. Maintenant, l'impact concret et politique et la réforme des programmes sont beaucoup plus modestes. Une idée qui a été vraiment reprise, ce sont les prisons ouvertes. Nous sommes en effet tombés au bon moment, l'idée a été reprise par la ministre de la Justice. C'est donc à peu près la première fois que grâce à notre action, une idée va être mise en œuvre. C'est une satisfaction, cette mise en œuvre, ce sujet, c'est quand même important. Concernant les grands sujets, ils circulent de façon certaine. J'ai d'ailleurs rencontré quasiment la moitié des ministres du gouvernement pour défendre les grandes thèses du think tank...

\section{Il y a une écoute quand même...}

Il y a donc une écoute et une curiosité, surtout avec le gouvernement actuel qui est plus réceptif que le précédent. Mais pour le moment cela ne va pas plus loin que cela. Nathalie KosciuskoMorizet est celle qui avait repris le plus d'éléments dans son programme; elle a été la seule qui, à un moment donné s'est rapprochée de nous et a transcrit un certain nombre de nos propositions dans son programme. Il s'agit d'une démarche 
assez rare et c'est la manière dont les choses devraient se passer : une adaptation des idées dans un programme. Nous ne nous en occupons pas directement. Cela nous permet d'être plus radicaux, plus tranchés, plus idéalistes parfois. C'était une bonne illustration de la manière dont cela doit fonctionner. Bon, comme vous voyez, l'impact réel reste quand même modeste.
$\mathrm{N} \cdot \mathrm{O} \cdot \mathrm{T} \cdot \mathrm{E} \cdot \mathrm{S}$

1. [NDLE] Le CATO Institute est un think tank américain d'obédience libertarienne fondé dans les années 1970 avec le concours de l'économiste anarcho-capitaliste Murray Rothbard.

2. Gaspard Koenig, Les aventuriers de la liberté, Paris, Plon, 2016. 


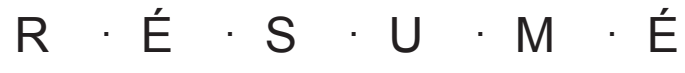

Comment s'engage-t-on pour les idées néo-libérales en France ? En tant que directeur du think tank GénérationLibre et philosophe engagé dans le débat public, Gaspard Koenig nous offre une vue de l'intérieur du mouvement néo-libéral en France. Les idées qui l'animent proviennent de ses lectures en philosophie politique davantage que de la lecture d'économistes théoriciens du marché. Son néo-libéralisme modéré se distingue de traditions concurrentes qui font de l'État la source de tous les maux de la société. Il s'inscrit, à l'inverse, dans une tradition libérale qui attribue à l'État un rôle d'arbitre s'assurant que chacun ait la capacité d'être autonome et de choisir son mode de vie. Dans cet entretien, il nous expose les raisons qui l'ont poussé à défendre et à créer un think tank, GénérationLibre, pour s'assurer de la promotion de ces idées (plutôt que des répertoires d'action concurrents). Il explique son fonctionnement et les raisons qui l'ont poussé à privilégier le registre de l'expertise par rapport à celui de l'« intellectuel total ». Il expose sa démarche inspirée de l'expérience des think tanks au Royaume-Uni et du penseur néo-libéral Friedrich Hayek, visant à convaincre un «public éclairé » composé d'intermédiaires de pensée. L'objectif est pour le philosophe de changer le climat d'opinion régnant dans la société en tenant un discours respecté par les élites. Pour ce faire, il privilégie la production d'expertise à disposition des décideurs publics, ainsi que l'établissement de réseaux de personnes partageant sa vision du libéralisme. Son témoignage révèle également que, si les mobilisations en faveur du néo-libéralisme se développent et revêtent des formes comparables à celles observées dans les pays qui ont donné naissance à cette idéologie, elles demeurent contraintes par plusieurs facteurs institutionnels propres au contexte français.

\section{Abstract}

How are we committed to neo-liberal ideas in France? As director of the think tank GénérationLibre and philosopher engaged in the public debate, Gaspard Koenig offers us a view from the inside of the neo-liberal movement in France. The ideas that animate him were more influenced by his readings in political philosophy than from his readings of economists theorists of the market. His moderate neo-liberalism stands out from competing traditions that make the state the source of all the ills of society. On the other hand, it is part of a liberal tradition that attributes to the state the role of arbiter, ensuring that everyone has the capacity to be autonomous and to choose their way of life. In this interview, he explains the reasons that led him to defend and create a think tank, GénérationLibre, to ensure the promotion of these ideas (rather than competing repertoire of contention). He explains how it works and the reasons that led him to favor the register of expertise compared to that of the "total intellectual". He exposes his approach inspired by the experience of think tanks in the United Kingdom and the neoliberal thinker Friedrich Hayek, aimed at convincing an "enlightened public" composed of intermediaries of thought. The goal is for the philosopher to change the climate of opinion prevailing in society by holding a discourse respected by the elites. To do this, it favors the production of expertise available to public decision makers, as well as the establishment of networks of people sharing its vision of liberalism. His testimony also reveals that, while the mobilizations in favor of neo-liberalism develop and take on forms comparable to those observed in the countries that gave birth to this ideology, they remain constrained by several institutional factions specific to the French context. 
